\title{
The utility of negative pressure wound therapy with instillation for wound bed preparation
}

This article was published in the following Dove Press journal:

Chronic Wound Care Management and Research

\section{Luis Fernández}

Department of General Surgery, University of Texas Health Science Center, Tyler, TX, USA
Correspondence: Luis Fernández Department of General Surgery, University of Texas Health Science Center, 1020 E. Idel Street, Tyler, TX, USA

Tel +903-535-2902

Email thebigkahunalI5@gmail.com

\begin{abstract}
Negative pressure wound therapy (NPWT) with instillation and dwell-time (NPWTi-d) is an adjunctive therapy that facilitates wound cleansing via the introduction of a topical wound solution followed by the application of subatmospheric pressure. The author presents two cases to evaluate the effectiveness of NPWTi-d in the cleansing of complex and contaminated postoperative wounds. Prior postoperative surgical wounds or seromas were subject to incision and drainage and were packed with either iodoform gauze or hypochlorous acid-impregnated gauze. Infected wounds were swabbed to assay wound-resident bacteria and discern pathogenicity and the applicability of antibiotics. For each case, NPWTi-d was administered to the surgical defect, instilling a predetermined volume of hypochlorous acid wound solution (VASHE ${ }^{\mathbb{R}}$; SteadMed Medical, Fort Worth, TX, USA) with a 20-min dwell time followed by $2 \mathrm{hrs}$ of NPWT at $-125 \mathrm{mmHg}$. Patient 1, a 76-yearold female, was readmitted to the hospital for large bowel obstruction and an infected groin wound. NPWTi-d was applied to the left groin wound, and after seven days, the wound edges were drawn closer together. At discharge, the wound had almost fully reepithelialized. The patient followed up in clinic 6 days later with a completely healed wound. No complications were reported 4 months post-NPWTi-d. Patient 2, a 63-year-old male, underwent an abdominal wall reconstruction to resolve a Grade III complex incisional hernia. Two months later, the patient presented with erythema and a seroma along the incision. The seroma was opened, and NPWTi-d was applied for 3 days. After developing healthy granulation tissue, patient 2 transitioned to conventional NPWT. The wound was fully healed at 4-month follow-up. In the complex wounds for these two patients, NPWTi-d yielded positive outcomes in cleansing contaminated tissue. Randomized, controlled clinical studies should be developed to compare the effectiveness of NPWTi-d on wound healing and patient outcomes versus standard irrigation modalities.
\end{abstract}

Keywords: negative pressure wound therapy, instillation, dwell time, hernia, hypochlorous acid

\section{Introduction}

Complex and chronic wound etiologies can be a difficult-to-manage economic burden to health care institutions, thereby presenting manifold pecuniary considerations with respect to patient care and wound management. ${ }^{1}$ Wounds designated as either complex or chronic adversely affect over 6 million patients in the United States, with nearly US\$50 billion spent on their treatment annually. ${ }^{2,3}$ The rapid surge in health care costs associated with the treatment of chronic wounds is inextricably linked to increases in the aging population, an elevated incidence of diabetes and global spikes in obesity. ${ }^{4-6}$ In addition, the prevalence of wound 
recalcitrance and chronicity mitigates productivity for patients and institutions alike and can elevate the incidence of disability. ${ }^{7}$ Complex wounds may exert a significant impact on patient outcomes, morbidity, mortality, and cost and lead to longer hospital stays. ${ }^{8,9}$ Therefore, it is incumbent for surgeons to be well-versed in all aspects of the care and management of patients with complex wounds and their comorbid conditions and to possess faculty in the use of novel wound care modalities that can enhance the reparative process.

In the last few decades, the proliferation of new strategies in the prevention, treatment and management of complex and chronic wounds has significantly improved the care of patients. The development of negative pressure wound therapy (NPWT), introduced commercially after the studies of Argenta and Morykwas in 1997, was a seminal turning point in the way physicians approached the care of complex/chronic wounds. ${ }^{10}$ NPWT creates an environment that promotes wound healing by preparing the wound bed for closure, reducing edema, promoting granulation tissue formation and perfusion, and removing exudate and infectious material. These mechanisms and other effects of NPWT have been evaluated by various experimental and clinical studies. ${ }^{10-17}$

However, surface cleansing of complex wounds in patients with a multiplicity of comorbid conditions may not be sufficient to remove tissue debris and infectious materials to prepare a healthy wound bed for efficient healing. Conventional NPWT possesses some limitations, as NPWT alone lacks efficacy in solubilizing devitalized tissue. Innovating conventional NPWT by means of coupling the instillation of topical wound solutions as an adjunctive modality may improve wound bed preparation in complex and/or contaminated wounds. NPWT with instillation and dwell-time (NPWTi-d) is an adjunctive therapy that facilitates wound bed cleansing and infectious material removal. When utilizing NPWTi-d, a topical wound solution is introduced into the wound bed and dwells for a user-specified predetermined period and is then removed during a cycle of NPWT. The clinical use of NPWTi-d appears to be gaining traction, evidenced by a growing body of practical and comparative clinical literature. ${ }^{18-21}$ The existing literature suggests that NPWTi-d may be used to prepare the wound to promote granulation tissue formation between surgical debridements and may be utilized in various wound types. ${ }^{21-24}$ Several best practice advisory panels have developed recommendations to better instruct the clinician in the most appropriate uses of NPWTi-d. ${ }^{23,24}$ Here, the author presents two cases to evaluate the effectiveness of NPWTi$\mathrm{d}$ in the cleansing of complex and contaminated postoperative wounds and/or surgical defects. The novelty resides in NPWTi-d to instill a topical, hypochlorous antimicrobial wound solution over complex wounds in a complicated wound location with an elevated risk of morbidity. This dictates the enlistment of a multidisciplinary and multimodal approach in an effort to address and manage factors that inhibit wound healing.

\section{Case presentations Case I}

A 76-year-old female with a medical history of deep venous thrombosis, esophagitis, shingles, and osteoporosis was admitted from the emergency department (ED) for dehydration and high-grade small bowel obstruction due to an incarcerated left inguinal hernia. The patient had been symptomatic for 6 days prior to admission. The patient underwent emergent surgical intervention with resection of $30 \mathrm{~cm}$ of strangulated small bowel noted within the femoral canal. A small bowel anastomosis was created, and the hernia defect was repaired with a $20 \mathrm{~cm} \times$ 25-cm synthetic mesh (XenMatrix ${ }^{\text {TM}}$; C.R. Bard, Inc., Murray Hill, NJ, USA) plug via a Lockwood's infrainguinal approach. The patient had an uneventful recovery and was discharged home on postoperative day 8 .

Approximately 2 weeks after her operation, the patient was readmitted with symptoms of partial large bowel obstruction from severe constipation, as well as a left groin wound infection. Hematological assays measured a white blood cell count of 18.4 and no bandemia, but the patient had some clinical signs of early sepsis. The left groin wound was opened and $10 \mathrm{cc}$ of pus was drained from the wound. Anaerobic and aerobic cultures were taken from the wound area, and the wound was packed open with iodoform gauze. The patient underwent placement of a peripherally inserted central catheter for nutritional optimization and colonic disimpaction with enemas. The patient was also placed on a 4-week therapeutic regimen of an intravenous antibiotic, piperacillin/tazobactam $\left(\right.$ ZOSYN $^{\circledR}$; Pfizer Inc., New York, NY, USA).

Local wound care was initiated with planned removal of the contaminated mesh at a later date. The patient continued to improve, and on hospital day (HD) 7, the author was consulted for possible mesh removal. The patient had been treated with wet-to-dry dressings up to this point and the 
patient did not appear toxic. In a subsequent hematology order, her WBC count measured 11.6, which was reduced relative to the initial measurement. The wound was inspected by the surgeon, and exposed mesh was visible (Figure 1A). NPWTi-d (V.A.C. VERAFLO ${ }^{\mathrm{TM}}$ Therapy System; KCI, an Acelity Company, San Antonio, TX, USA) was administered to the surgical defect, instilling hypochlorous acid wound solution $\left(\mathrm{VASHE}^{\mathbb{B}}\right.$; SteadMed Medical, Fort Worth, TX, USA) with a 20-min dwell time followed by $2 \mathrm{hrs}$ of NPWT at $-125 \mathrm{mmHg}$ (Figure 1B).

The patient progressively improved and was discharged home on HD 28. The wound had near complete epithelialization on the date of discharge (Figure 1C). The patient was followed up in clinic 6 days after discharge after completing 4 weeks of broad spectrum, intravenous antibiotics. The patient was asymptomatic with a completely healed wound and no recurrence of infection or hernia (Figure 1D). At 4 months post-NPWTi-d, the patient has no reported wound complications.

\section{Case 2}

The patient was a 63-year-old male, who had undergone extensive abdominal wall reconstruction for recurrent complex ventral hernia 2 months prior to admission. The patient had a history of bilateral inguinal hernia repair with right laparoscopic synthetic inguinal hernia mesh failure, small bowel obstruction, resulting in exploratory celiotomy with appendectomy and subsequent repair with explantation of contaminated synthetic mesh, and intraperitoneal repair of right inguinal hernia with biologic dermal mesh (STRATTICETM
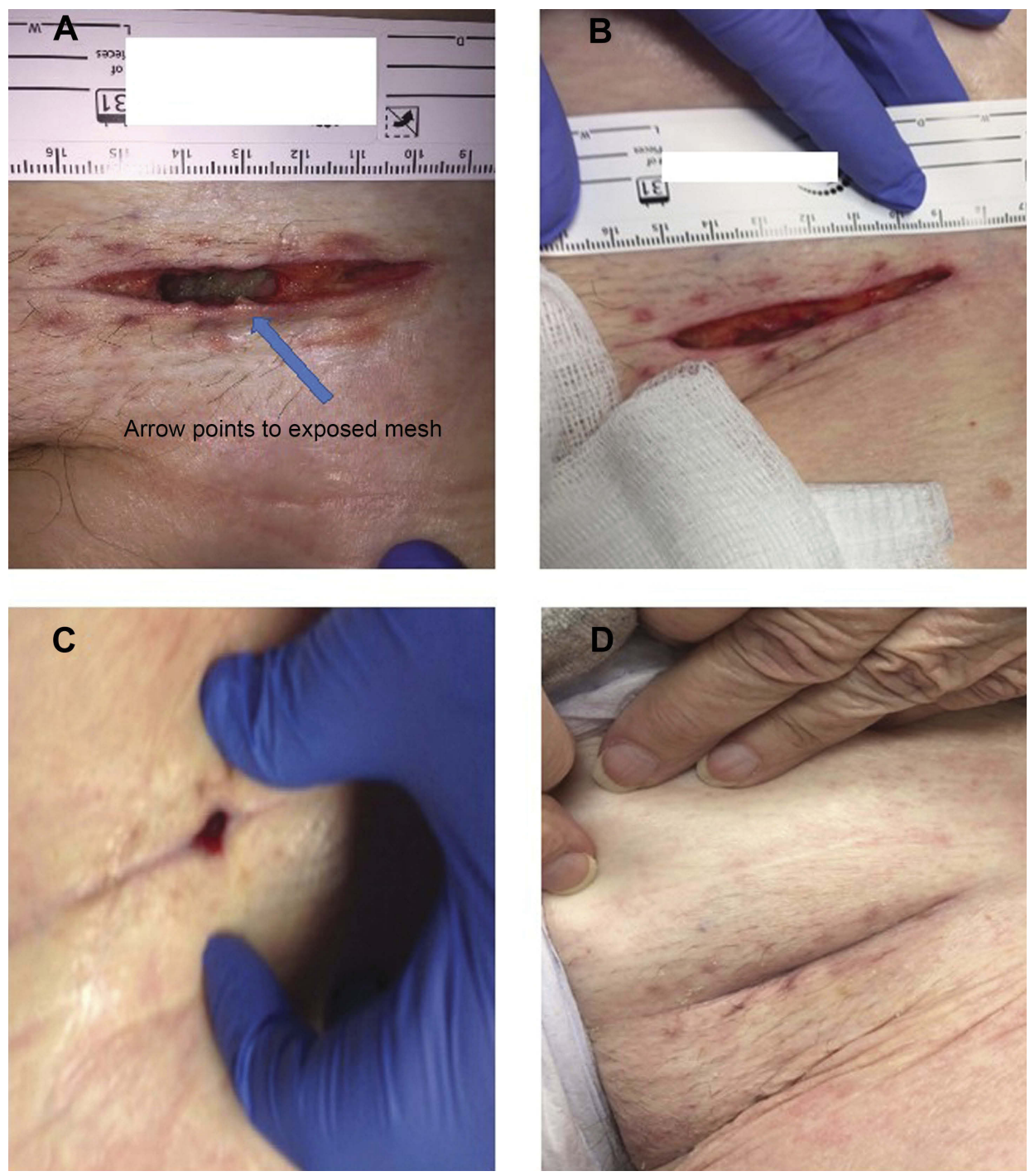

Figure I (A) Left groin wound at inspection reveals partial exposure of the contaminated synthetic mesh. (B) At day 7 of NPWTi-d, wound edges are being drawn together. (C) Left groin wound at day 15 of NPWTi-d exhibits near complete epithelialization. (D) Left groin wound is completely healed 6 days postdischarge. 
Reconstructive Tissue Matrix; Allergan plc, Dublin, Ireland). The patient had previously undergone damage control surgery and an open abdomen for intraperitoneal abscess. He had a primary fascial closure at that time.

He also had a history of prior wound infection and a right scrotal ischemia with resultant loss of the right testicle; he subsequently developed a large recurrent incisional hernia (Figure 2A and B). Patient comorbidities included: COPD, tobacco use, morbid obesity with planned recent weight loss, and schizophrenia. COPD was managed with the bronchodilator, tiotropium bromide (Spiriva ${ }^{\circledR}$; Boehringer Ingelheim Pharmaceuticals, Inc.; Ridgefield, CT, USA) and a rescue inhaler. The patient had a Grade III complex incisional hernia as defined by Ventral Hernia Working Group hernia grading system (Figure 2A and B). ${ }^{25,26}$

The index surgery consisted of exploratory celiotomy, extensive adhesiolysis, $360^{\circ}$ myocutaneous flap advancement, placement of $20 \times 25-\mathrm{cm}$ biologic dermal mesh (STRATTICE ${ }^{\text {TM }}$ Reconstructive Tissue Matrix; Allergan plc, Dublin, Ireland) underlaid with partial component separation of the anterior abdominal wall and incisional washout with hypochlorous acid wound solution instillation $\left(\mathrm{VASHE}^{\circledR}\right.$; SteadMed Medical, Fort Worth, TX, USA) in the intraperitoneal and subcutaneous space. A mechanical bioresorbable adhesion barrier (Seprafilm ${ }^{\circledR}$; Sanofi-Aventis US LLC., Bridgewater, NJ, USA) was placed within the peritoneal cavity to mitigate the extent of postoperative adhesions.

Drains were placed beneath the subcutaneous flaps, the skin was sutured and closed incision negative pressure therapy (PREVENA ${ }^{\mathrm{TM}}$ Incision Management System with PREVENA $^{\mathrm{TM}}$ Customizable $^{\mathrm{TM}}$ Dressing; KCI, an Acelity Company, San Antonio, TX, USA) was applied over the closed incision (Figure 3A and B). At a 3-week postoperative follow-up in our clinic, the patient had some superficial skin ischemia without incisional dehiscence but was otherwise asymptomatic (Figure 3C). His drains were removed, and the patient was scheduled for follow-up in 2 weeks.

Two months from his abdominal wall reconstruction, the patient presented to the ED with erythema and a seroma of the lower aspect of the wound (Figure 4A). Hematological examination revealed that the patient had a WBC count of 8 and no bandemia, and the patient appeared nontoxic. The seroma was opened, and $200 \mathrm{cc}$ of non-malodorous serous fluid was evacuated. The wound was cleansed with hypochlorous acid wound solution (VASHE $^{\circledR}$; SteadMed Medical, Fort Worth, TX, USA)
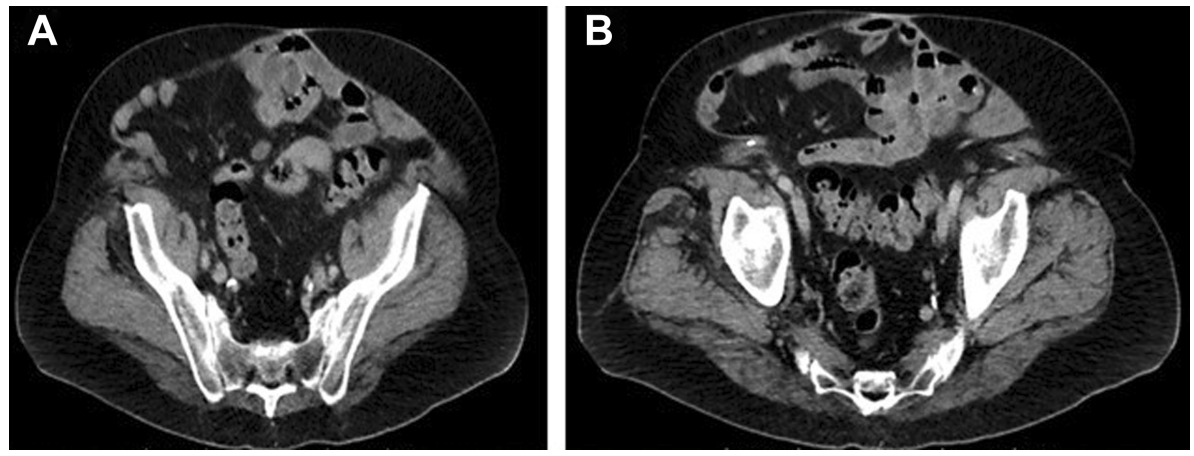

Figure 2 (A) Transverse CT scan of the abdominopelvic region reveals the development of a large incisional hernia. (B) Transverse CT scan of the abdominopelvic region ascertained a diagnosis of a Grade III complex incisional hernia as defined by the VHWG hernia grading system.
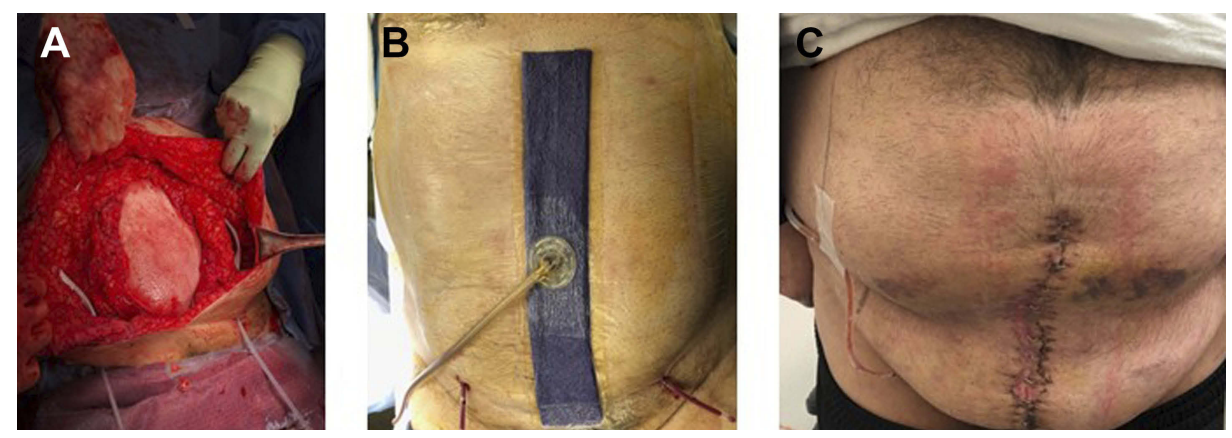

Figure 3 (A) Patient underwent index surgery for biologic dermal mesh placement and the transient installation of drains beneath subcutaneous flaps. (B) ciNPT was applied to the closed incision. (C) At 3-week postoperative follow-up, incision exhibited superficial skin ischemia, but the patient was asymptomatic. 

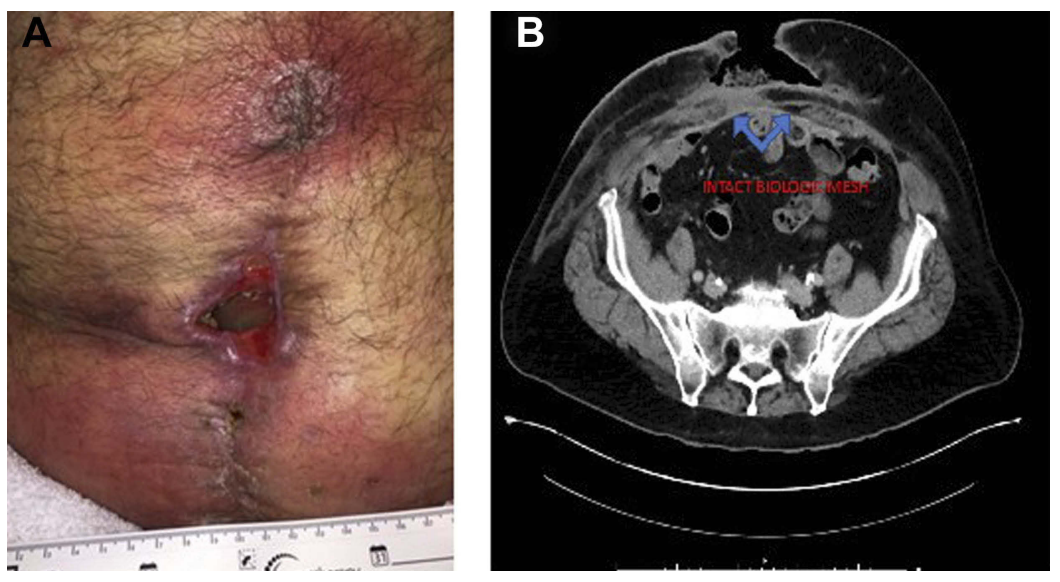

Figure 4 (A) Patient presented to the ED with erythema and seroma at the inferior aspect of the dehisced incision. (B) Transverse CT scan of the abdominopelvic region reveals the presence of seroma and intact biologic dermal mesh.

and then packed with woven gauze bandage impregnated with hypochlorous acid wound solution VASHE $^{\circledR}$; SteadMed Medical, Fort Worth, TX, USA).

An abdominal CT scan ascertained that the underlying biologic dermal mesh (STRATTICE ${ }^{\mathrm{TM}}$ Reconstructive Tissue Matrix (RTM); Allergan plc, Dublin, Ireland) was intact (Figure 4B). As the biologic mesh had been positioned intraperitoneally as a retromuscular underlay, the abdominal fascia was closed where the biologic mesh was placed. Then, a nonadhering silicone dressing (ADAPTIC TOUCH $^{\text {TM }}$, KCI, an ACELITY Company, San Antonio, TX, USA) was applied over the closed fascia.

For 3 days, NPWTi-d (V.A.C. VERAFLO ${ }^{\mathrm{TM}}$ Therapy System; KCI, an Acelity Company, San Antonio, TX, USA) was administered to the surgical defect, instilling hypochlorous acid wound solution $\left(\mathrm{VASHE}^{\circledR}\right.$; SteadMed Medical, Fort Worth, TX, USA) with a 20-min dwell time, followed by $2 \mathrm{hrs}$ of NPWT at $-125 \mathrm{mmHg}$. The topical wound solution did not come into contact with the mesh placed under the fascia. (The manufacturer warns that
NPWTi-d is not intended for use with cellular or acellular bioengineered tissues, such as STRATTICETM RTM.) The wound demonstrated significant improvement evidenced by the development of healthy granulation tissue and a reduction in erythema (Figure 5A). The patient was subsequently transitioned to conventional NPWT (V.A.C. ${ }^{\circledR}$ Therapy System; KCI, an Acelity Company, San Antonio, TX, USA) for outpatient management (Figure 5B). At a 4-month clinic follow-up, the patient's wound was fully healed, and the patient was asymptomatic (Figure 5C).

\section{Discussion}

There are over 6 million individuals in the United States who suffer from complex and chronic wounds. The estimated cost of care of these patients is nearly US\$50 billion annually. $^{2-5}$ Through diligent work by dedicated researchers, the management of acute and chronic wounds has improved in the last 2 decades. Novel approaches in prevention, nutritional assessment, treatment management and innovative technologies have revolutionized the care
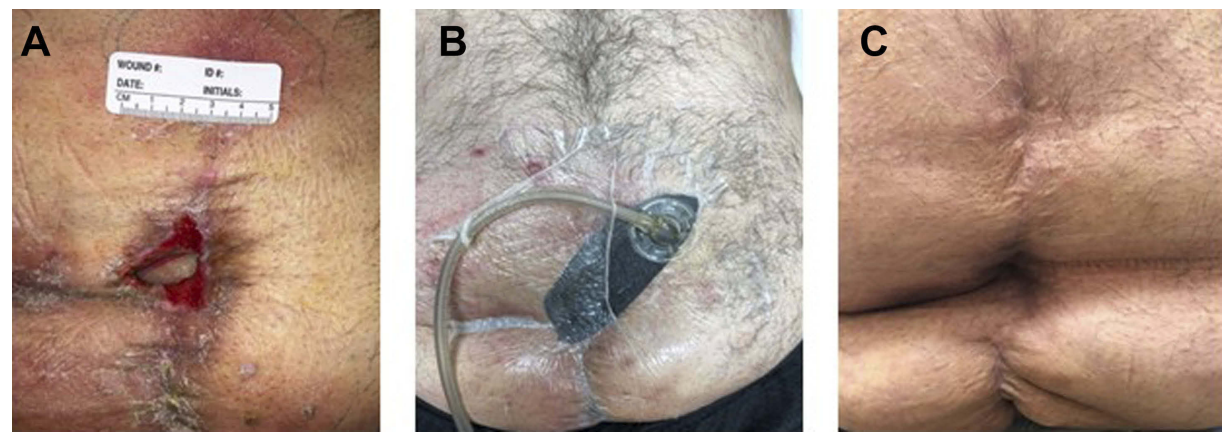

Figure 5 (A) Wound exhibits the presence of granulation tissue after 3 days of NPWTi-d. (B) NPWTi-d was discontinued and the patient transitioned to conventional NPWT for outpatient management. (C) Wound at 4-month clinical follow-up is fully healed. 
of these difficult patients. The successful application of NPWTi-d represents a paradigm shift in the way clinicians approach the care of the acute/complex/chronic wound. ${ }^{10}$ NPWTi-d promotes wound healing by cleansing the wound bed, removing exudate and infectious material, reducing local edema, promoting perfusion and granulation tissue formation. These mechanisms create an environment that enhances overall healing and prepares the wound for closure. These findings have been documented by various reports in the literature. ${ }^{10-17}$

Infected wounds continue to be a difficult challenge particularly in postoperative patients and remain a major component of reported hospital nosocomial infection rates. ${ }^{27}$ Wounds are quickly colonized with adherent bacterial glycocalyx (biofilm) after initial exposure and biofilm's detrimental role in the wound-healing process has been described. ${ }^{28,29}$ These colonized wounds require an intensive multimodal approach for healing. Meticulous attention to nutrition, immunocompetence, control of comorbid conditions, judicious use of antibiotics, and effective surgical debridement when indicated are the foundation of successful management. The optimal treatment paradigm in preparation of the wound bed should be that which is the most efficient in removing barriers to wound healing (eg, elevated inflammatory cytokines, cellular debris, and infectious material). Automated wound cleansing via NPWTi-d may broaden the clinician's options in the management of complex wounds. NPWTi$\mathrm{d}$, the next evolutionary step in adjunctive modalities employing subatmospheric pressure, has been demonstrated to be effective in cleansing complex infected wounds. ${ }^{4,19-21,23}$

In vivo evidence has demonstrated in a porcine model that noninfected, full-thickness wounds treated with NPWTi-d (normal saline and use of a reticulated opencell foam [ROCF-V; V.A.C. VERAFLO ${ }^{\mathrm{TM}}$ Dressing; KCI, an Acelity Company, San Antonio, TX, USA] specifically designed for instillation therapy) resulted in increased formation of granulation tissue after 7 days relative to conventional NPWT with the standard ROCF dressing (V.A.C. ${ }^{\circledR}$ GRANUFOAM ${ }^{\mathrm{TM}}$ Dressing; KCI, an Acelity Company, San Antonio, TX, USA). ${ }^{30}$ NPWTi-d provides discrete wound irrigation and fluid removal phases between cycles in which subatmospheric pressure is applied, thereby simplifying wound cleansing via the automated distribution of a predetermined volume of solution to the wound bed. ${ }^{30}$ In comparison to the ROCF dressings traditionally used for NPWT, the ROCF-V exhibits improved hydrophobic and mechanical properties, which enhances fluid delivery to the wound bed while potentially mitigating the likelihood of foam tearing or the retention of foam residue at dressing changes. ${ }^{30}$

In a similar porcine model, Lessing et $\mathrm{al}^{31}$ employed NPWTi-d instilling normal saline and compared it to conventional NPWT that applied either intermittent (alternating between pressure and no pressure) or variable (programmed transitions between high and low pressure) modes of subatmospheric pressure. At day 7, they noted that the average observed granulation tissue was not statistically different between the diverse modes of subatmospheric pressure applied. ${ }^{31}$ However, they did report that the average thickness of granulation tissue within NPWTid-treated wounds was statistically greater $(P<0.05)$ by $44 \%, 57 \%$, and $40 \%$ in conventional NPWT-treated wounds where continuous, intermittent, and variable subatmospheric pressure were applied, respectively. ${ }^{31}$

In a preclinical investigation to evaluate the extent of tissue damage, cleansing, and potential of crosscontamination during wound cleansing and application of NPWTi-d demonstrated advantages over both lowpressure and high-pressure lavage of wounds. ${ }^{32}$ Using a porcine-derived ex vivo wound model, scanning electron microscopy revealed increased observable tissue trauma stemming from lavage treatment at low and high pressures relative to NPWTi-d, with the extent of the trauma corresponding to the pressure exerted by the irrigant. ${ }^{32}$ Additionally, three-dimensional photography evaluated tissue damage by measuring immediate tissue distension and demonstrated significantly greater $(P<$ 0.05 ) swelling in wounds treated with low-pressure lavage compared to wounds treated with NPWTi-d. ${ }^{32}$

In a separate study, the inoculation of benchtop wound models with fluorescent bacterial particles was enlisted to assess the potential for environmental cross-contamination during low-pressure lavage and NPWTi-d. ${ }^{32}$ While no evidence of cross-contamination was noted following treatment with NPWTi-d, Allen et $\mathrm{al}^{32}$ did report onehalf of the particles were aerosolized during low-pressure lavage $(P<0.05)$.

In Case 1 of this study, the synthetic mesh was contaminated. Explantation of contaminated mesh requires technical considerations by the surgical team; however, this was avoided in this instance by enlisting NPWTi-d to cleanse the surgical defect. The contaminated nature of the surgical defects in both cases required effective detersion of the prepared wound bed, which was provided by NPWTi-d 
through the instillation of a topical wound solution. While studies have demonstrated that normal saline is as effective as polyhexanide-betaine, a topical hypochlorous acid wound solution was used as an instillation solution for its lack of cytotoxicity and antimicrobial effect. ${ }^{28}$

The limitations of this study include those inherent to a brief case report, such as the sample size and the lack of robust statistical analysis on the cost-effectiveness of durable medical equipment that applies subatmospheric pressure. Clarity is also needed as to whether instillation as an adjunctive to NPWT imparts any incremental benefit that conventional NPWT alone is unable to afford in facilitating positive wound healing outcomes. Whether there is any incremental effect of NPWTi-d relative to NPWT warrants investigation.

\section{Conclusion}

In the complex and contaminated wounds for these two patients, NPWTi-d demonstrated a marked and positive effect in the cleansing of the wound and the removal of exudate and cellular debris. It also facilitated the rapid creation of granulation tissue to effectively create a healthier wound bed. As experience with this approach expands, we will have better data that will help inform the clinician as to which therapeutic regimen is most appropriate for the treatment of complex and/or contaminated wounds. Randomized, controlled clinical studies should be developed to compare the effectiveness of NPWTi-d to other, non-NPWT methods of care and to determine the effectiveness of NPWTi-d versus standard irrigation practices on wound healing and overall patient outcomes.

\section{Consent Statement}

Written informed consent was acquired from the patients in order to sanction the inclusion of their respective case details and the associated images published for this case series. Institutional approval was not required to publish the case details.

\section{Acknowledgment}

Willie M. Heard III, PhD, (KCI, an Acelity Company) provided medical writing support.

\section{Disclosure}

Dr Fernández is a consultant to $\mathrm{KCI}$, an Acelity Company. He is also a speaker/lecturer for the company. The author reports no other conflicts of interest in this work.

\section{References}

1. Jarbrink K, Ni G, Sonnergren H, et al. The humanistic and economic burden of chronic wounds: a protocol for a systematic review. Syst Rev. 2017;6(1):15. doi:10.1186/s13643-016-0400-8

2. Fife CE, Carter MJ, Walker D, Thomson B. Wound care outcomes and associated cost among patients treated in US outpatient wound centers: data from the US wound registry. Wounds. 2012;24 (1):10-17.

3. Driver VR, Fabbi M, Lavery LA, Gibbons G. The costs of diabetic foot: the economic case for the limb salvage team. $J$ Am Podiatr Med Assoc. 2010;100(5):335-341.

4. Hossain P, Kawar B, El Nahas M. Obesity and diabetes in the developing world - a growing challenge. $N$ Engl J Med. 2007;356 (3):213-215. doi:10.1056/NEJMp068177

5. Administration for Community Living. 2017 profile of older Americans; 2018. Available from: https://wwwaclgov/[serialonline]. Administration for Community Living. Accessed August 29, 2018.

6. Singer AJ, Clark RA. Cutaneous wound healing. $N$ Engl J Med 1999;341(10):738-746. doi:10.1056/NEJM199909023411006

7. Nussbaum SR, Carter MJ, Fife CE, et al. An economic evaluation of the impact, cost, and medicare policy implications of chronic nonhealing wounds. Value Health. 2018;21(1):27-32. doi:10.1016/j. jval.2017.07.007

8. Ferreira MC, Tuma Junior P, Carvalho VF, Kamamoto F. Complex wounds. Clinics. 2006;61(6):571-578.

9. Kirkland KB, Briggs JP, Trivette SL, Wilkinson WE, Sexton DJ. The impact of surgical-site infections in the 1990s: attributable mortality, excess length of hospitalization, and extra costs. Infect Control Hosp Epidemiol. 1999;20(11):725-730. doi:10.1086/501572

10. Argenta LC, Morykwas MJ. Vacuum-assisted closure: a new method for wound control and treatment: clinical experience. Ann Plast Surg. 1997;38(6):563-576

11. Saxena V, Hwang CW, Huang S, Eichbaum Q, Ingber D, Orgill DP. Vacuum-assisted closure: microdeformations of wounds and cell proliferation. Plast Reconstr Surg. 2004;114(5):1086-1096.

12. McNulty AK, Schmidt M, Feeley T, Kieswetter K. Effects of negative pressure wound therapy on fibroblast viability, chemotactic signaling, and proliferation in a provisional wound (fibrin) matrix. Wound Repair Regen. 2007;15(6):838-846. doi:10.1111/j.1524475X.2007.00287.x

13. McNulty AK, Schmidt M, Feeley T, Villanueva P, Kieswetter K. Effects of negative pressure wound therapy on cellular energetics in fibroblasts grown in a provisional wound (fibrin) matrix. Wound Repair Regen. 2009;17(2):192-199. doi:10.1111/j.1524475X.2009.00460.x

14. Scherer SS, Pietramaggiori G, Mathews JC, Prsa MJ, Huang S, Orgill DP. The mechanism of action of the vacuum-assisted closure device. Plast Reconstr Surg. 2008;122(3):786-797. doi:10.1097/ PRS.0b013e31818237ac

15. Dastouri P, Helm DL, Scherer SS, Pietramaggiori G, Younan G, Orgill DP. Waveform modulation of negative-pressure wound therapy in the murine model. Plast Reconstr Surg. 2011;127(4):1460-1466. doi:10.1097/PRS.0b013e31820a63cb

16. Armstrong DG, Lavery LA. Diabetic foot study consortium. negative pressure wound therapy after partial diabetic foot amputation: a multicentre, randomised controlled trial. Lancet. 2005;366 (9498):1704-1710. doi:10.1016/S0140-6736(05)67695-7

17. Blume PA. Comparison of negative pressure wound therapy using vacuum-assisted closure with advanced moist wound therapy in the treatment of diabetic foot ulcers: a multicenter randomized controlled trial: response to hemkens and waltering. Diabetes Care. 2008;31 (10):e77. doi: $10.2337 / \mathrm{dc} 07-1868$

18. Kim PJ, Attinger CE, Steinberg JS, Evans KK. Negative pressure wound therapy with instillation: past, present, and future. Surg Technol Int. 2015;26:51-56. 
19. Kim PJ, Attinger CE, Crist BD, et al. Negative pressure wound therapy with instillation: review of evidence and recommendations. Wounds. 2015;27(12):S2-S19.

20. Gabriel A, Kahn K, Karmy-Jones R. Use of negative pressure wound therapy with automated, volumetric instillation for the treatment of extremity and trunk wounds: clinical outcomes and potential cost-effectiveness. Eplasty. 2014;14:e41.

21. Kim PJ, Attinger CE, Steinberg JS, et al. The impact of negative-pressure wound therapy with instillation compared with standard negative-pressure wound therapy: a retrospective, historical, cohort, controlled study. Plast Reconstr Surg. 2014;133(3):709-716. doi:10.1097/01.prs.0000438060.46290.7a

22. Huang C, Leavitt T, Bayer LR, Orgill DP. Effect of negative pressure wound therapy on wound healing. Curr Probl Surg. 2014;51 (7):301-331. doi:10.1067/j.cpsurg.2014.04.001

23. Gupta S, Gabriel A, Lantis J, Teot L. Clinical recommendations and practical guide for negative pressure wound therapy with instillation. Int Wound J. 2016;13(2):159-174. doi:10.1111/iwj.12452

24. McKanna M, Geraci J, Hall K, et al. Clinician panel recommendations for use of negative pressure wound therapy with instillation. Ostomy Wound Manage. 2016;62(4):S3-S14.

25. Breuing $\mathrm{K}$, Breuing $\mathrm{K}$, Butler $\mathrm{CE}$, et al. Incisional ventral hernias: review of the literature and recommendations regarding the grading and technique of repair. Surgery. 2010;148(3):544-558. doi:10.1016/ j.surg.2010.01.008
26. Guerra O, Maclin MM. Non-crosslinked porcine-derived acellular dermal matrix for the management of complex ventral abdominal wall hernias: a report of 45 cases. Hernia. 2014;18(1):71-79. doi:10.1007/s10029-013-1148-x

27. Sen CK, Gordillo GM, Roy S, et al. Human skin wounds: a major and snowballing threat to public health and the economy. Wound Repair Regen. 2009;17(6):763-771. doi:10.1111/j.1524-475X.2009.00543.x

28. Niezgoda JA, Sordi PJ, Hermans MH. Evaluation of vashe wound therapy in the clinical management of patients with chronic wounds. Adv Skin Wound Care. 2010;23(8):352-357. doi:10.1097/01. ASW.0000383198.35815.a2

29. Prakash B, Veeregowda BM, Krishnappa G. Biofilms: a survival strategy of bacteria. Curr Sci. 2003;85(9):1299-1307.

30. Lessing C, Slack P, Hong KZ, Kilpadi D, McNulty A. Negative pressure wound therapy with controlled saline instillation (NPWTi): dressing properties and granulation response in vivo. Wounds. 2011;23(10):309-319.

31. Lessing MC, James RB, Ingram SC. Comparison of the effects of different negative pressure wound therapy modes-continuous, noncontinuous, and with instillation-on porcine excisional wounds. Eplasty. 2013;13:e51.

32. Allen D, LaBarbera LA, Bondre IL, et al. Comparison of tissue damage, cleansing and cross-contamination potential during wound cleansing via two methods: lavage and negative pressure wound therapy with instillation. Int Wound J. 2014;11(2):198-209. doi:10.1111/j.1742-481X.2012.01073.x
Chronic Wound Care Management and Research

\section{Publish your work in this journal}

Chronic Wound Care Management and Research is an international, peer reviewed, open access, online journal publishing original research, reviews, editorials, and commentaries on the causes and management of chronic wounds and the major issues related to chronic wound management. Topics also include chronic wounds as comorbidities to other conditions, patient adherence to therapy, and the economic burden of chronic wounds. The manuscript management system is completely online and includes a very quick and fair peer review system, which is all easy to use. Visit http://www.dovepress.com/ testimonials.php to read real quotes from published authors. 